\title{
The clinical value of carcinoembryonic antigen for tumor metastasis assessment in lung cancer
}

\author{
Jiasi Wang ${ }^{\text {Equal first author, } 1}$, Yanpeng Chu ${ }^{\text {Equal first author, } 2}{ }^{2}, \mathbf{J i e ~ L i ~}^{2}$, Tinjie Wang ${ }^{1}$, Liangli Sun ${ }^{1}$, Pingfei Wang ${ }^{2}$, Xiangdong \\ Fang $^{3}$, Fanwei Zeng ${ }^{2}$, Junfeng Wang ${ }^{\text {Corresp., } 4}$, Fanxin Zeng ${ }^{\text {Corresp. } 2,3}$ \\ ${ }^{1}$ Department of Clinical laboratory, Dazhou Central Hospital, Dazhou, Sichuan, China \\ 2 Department of Clinical Research Center, Dazhou Central Hospital, Dazhou, Sichuan, China \\ 3 Department of Oncology, Dazhou Central Hospital, Dazhou, Sichuan, China \\ 4 Department of Biomedical Data Sciences, Leiden University Medical Center, Leiden, The Netherlands \\ Corresponding Authors: Junfeng Wang, Fanxin Zeng \\ Email address: j.wang@lumc.nl, zengfx@pku.edu.cn
}

Background: Carcinoembryonic antigen (CEA) as a diagnostic or prognostic marker has been widely studied in patients with lung cancer. However, the relationship between serum CEA and tumor metastasis in lung cancer remains controversial. This study aimed to investigate the ability of serum CEA to assess tumor metastasis in lung cancer patients. Methods: We performed a retrospective analysis of 238 patients diagnosed with lung cancer from January to December 2016 at pneumology department of Dazhou Central Hospital (Dazhou, China). Serum CEA levels were quantified in each patient at the time of diagnosis of lung cancer. Metastasis was confirmed by computed tomography (CT), and/or positron emission tomography (PET) and/or surgery or other necessary detecting methods.

Results: Of the 213 patients eligible for final analysis, 128 were diagnosed with metastasis and 85 were diagnosed without metastasis. Compared to non-metastatic patients, the serum CEA was markedly higher in patients with metastasis $(p<0.001)$, and the area under the curve (AUC) was 0.724 (95\% confidence interval [Cl], 0.654-0.793). Subsequent analyses regarding the number and location of tumor metastases showed that CEA also had clinical value for multiple metastases versus single metastasis ( $A U C=0.780$, $95 \% \mathrm{Cl}$ 0.699-0.862) and distant metastasis versus non-distant metastasis ( $A U C=0.815$, $95 \% \mathrm{Cl}$ 0.733-0.897). In addition, we found that tumor size, histology diagnosis, age and gender had no impact on the assessment performance of CEA. Conclusion: Our study suggested the serum CEA as a valuable marker for tumor metastases assessment in newly diagnosed lung cancer patients, which could have some implications in clinical application. 
1 The clinical value of carcinoembryonic antigen for tumor metastasis assessment in lung

2 cancer

3 Jiasi Wang ${ }^{1, *}$, Yanpeng $\mathrm{Chu}^{2, *}$, Jie Li², Tingjie Wang ${ }^{1}$, Liangli Sun ${ }^{1}$, Pingfei Wang ${ }^{2}$, Xiangdong

4 Fang ${ }^{3}$, Fanwei Zeng ${ }^{2}$, Junfeng Wang, \#, Fanxin Zeng 2, 3, \#

$5 \quad{ }^{1}$ Department of Clinical laboratory, Dazhou Central Hospital, Dazhou, Sichuan, China

6 2Department of Clinical Research Center, Dazhou Central Hospital, Dazhou, Sichuan, China

$7 \quad{ }^{3}$ Department of Oncology, Dazhou Central Hospital, Dazhou, Sichuan, China

$8{ }^{4}$ Department of Biomedical Data Sciences, Leiden University Medical Center, Leiden, The

$9 \quad$ Netherlands

$11{ }^{*}$ These authors contributed equally

$12{ }^{\#}$ Corresponding Authors:

13 Fanxin Zeng 2,3

14 No.56 Nanyuemiao Street, Tongchuan District Dazhou, Sichuan province, P. R. China

15 Email address: zengfx@pku.edu.cn

16 Junfeng Wang 4

17 Secretariaat G4-Noord, Meibergdreef 9, Amsterdam, Netherlands

18 Email address: j.wang@,lumc.nl 


\section{Abstract}

20 Background: Carcinoembryonic antigen (CEA) as a diagnostic or prognostic marker has been widely studied in patients with lung cancer. However, the relationship between serum CEA and tumor metastasis in lung cancer remains controversial. This study aimed to investigate the ability of serum CEA to assess tumor metastasis in lung cancer patients. Methods: We performed a retrospective analysis of 238 patients diagnosed with lung cancer from January to December 2016 at pneumology department of Dazhou Central Hospital (Dazhou, China). Serum CEA levels were quantified in each patient at the time of diagnosis of lung cancer. Metastasis was confirmed by computed tomography (CT), and/or positron emission tomography (PET) and/or surgery or other necessary detecting methods. Results: Of the 213 patients eligible for final analysis, 128 were diagnosed with metastasis and 85 were diagnosed without metastasis. Compared to non-metastatic patients, the serum CEA was markedly higher in patients with metastasis $(p<0.001)$, and the area under the curve (AUC) was 0.724 (95\% confidence interval [CI], 0.654-0.793). Subsequent analyses regarding the number and location of tumor metastases showed that CEA also had clinical value for multiple metastases versus single metastasis $(\mathrm{AUC}=0.780,95 \% \mathrm{CI} 0.699-0.862)$ and distant metastasis versus non-distant metastasis $(\mathrm{AUC}=0.815,95 \% \mathrm{CI} 0.733-0.897)$. In addition, we found that tumor size, histology diagnosis, age and gender had no impact on the assessment performance of CEA. Conclusion: Our study suggested the serum CEA as a valuable marker for tumor metastases assessment in newly diagnosed lung cancer patients, which could have some implications in clinical application. 
40

41

42

43

44

45

46

47

48

49

50

51

52

53

54

55

56 57 prognosis of patients.

58

59

60

\section{Introduction}

Lung cancer is the most common cause of death among all cancers worldwide (Torre et al. 2015; Wang et al. 2018).The overall survival rate depends on the stage of lung cancer, and patients with advanced lung cancer commonly have poor prognosis (Langer et al. 2010). Unfortunately, up to $70 \%$ of patients are already in advanced stage at the time of diagnosis (Arrieta et al. 2009; Patz et al. 2007). Evidence suggests that tumor metastasis reflects the relatively advanced stage of lung cancer and is responsible for more than $70 \%$ of patient deaths (Langer et al. 2010). The two main types of lung cancer are small-cell lung cancer (SCLC) and non-small-cell lung cancer (NSCLC), and the latter consists of three subtypes: adenocarcinoma, squamous cell carcinoma and large-cell carcinoma (Zheng 2016). In NSCLC patients, the main locations for tumor metastasis are lymph gland, bone, brain and liver (Wood et al. 2014). In NSCLC patients with no more than five metastases, systemic therapy, high-dose fractional radiotherapy or surgical removal of all affected sites can result in no progress in 3 years in 13\% of patients, even in stage IV patients, who can benefit from radical therapy. At the same time, minimally invasive surgery, palliative surgery, radiotherapy and bone modifier therapy can create better prognosis in NSCLC metastasis (Reck et al. 2014). Therefore, early detection of the presence and location of metastasis is helpful for choosing treatment methods and to improve the

58 Clinically, metastasis is confirmed by using a combination of clinical symptoms and imaging evidence when complete pathological evidence is available for lung cancer diagnosis (Gaga et al. 2013). The most commonly employed imaging modalities include computed 
61

62

63

64

tomography (CT), fused-positron-emitting-tomography-CT (PET-CT), magnetic-resonanceimaging (MRI) and chest X-ray (CXR) (Reck et al. 2014). However, the high expense of inspection and other factors may cause a great burden on the patient that could hinder the clinical monitoring and early detection of lung cancer metastasis (Patz et al. 2007; Zhang et al. 2017). Which, to a certain extent, limits the choice of treatment and negatively affects the prognosis of progressive cancer patients. In addition, patients can have metastases in certain areas, without obvious clinical symptoms, which are often ignored by both patients and doctors. Therefore, diagnostic techniques that are both economic and uncomplicated are urgently needed in clinics to indicate whether tumor metastases have occurred, which could guide doctors on whether a lung cancer patient with symptoms of suspected metastasis need more detailed examination. Blood-based biomarkers can be accessed easily, quickly, and economically, so they have the potential to greatly improve the efficiency of assessment. Carcinoembryonic antigen (CEA) is a glycoprotein involved in cell adhesion, which was first found in 1965 in the blood of patients with colon cancer (Crone-Munzebrock \& Carl 1990). Previous studies have reported that CEA could influence either epithelial cells or the surrounding stromal cells and immunity to alter related signaling programs such as TGF- $\beta \mathrm{R} 1$, apoptosis regulating proteins, $\mathrm{PI} 3 \mathrm{~K}$ and AKT activities to support metastasis progression in colorectal cancer patients (Beauchemin \& Arabzadeh 2013). The level of serum CEA expression and the trend of its changes in the treatment process have been validated by several studies on the clinical value of assessing the efficacy and prognosis of NSCLC (Ardizzoni et al. 2006; Arrieta et al. 2013). High levels of tumor markers at baseline, such as CEA and CA125, are correlated with worse survival in stage 
82

83

III-IV NSCLC patients (Cedres et al. 2011). Additionally, some studies have revealed that increased CEA levels were associated with increased risk of developing brain metastasis in patients with advanced NSCLC (Wang et al. 2017). However, many studies have reported that the prognostic value of CEA for lung cancer patients was ambiguous (Tsoukalas et al. 2017). Therefore, further studies are required for the diagnostic value of testing serum CEA in lung cancer patients.

We retrospectively analyzed the relationship between CEA and tumor metastasis in newly diagnosed patients with NSCLC and SCLC, to investigate the relationship between CEA and tumor metastasis, and assess whether CEA has certain clinical guiding value for organs involved and the number of organs involved in tumor metastasis.

\section{Materials and Methods}

\section{Study cohort}

We retrospectively reviewed 238 patients with a diagnosis of lung tumors from January to December 2016 at the pneumology department of Dazhou Central Hospital in this study. Clinical information, including gender, age, metastatic locations, histological type and tumor size was retrospectively obtained from electronic medical records. All these patients underwent fiberoptic bronchoscopy biopsy at the initial stage of cancer diagnosis after hospitalization. The specimens needed for pathological diagnosis came from fiberoptic bronchoscopy, percutaneous lung biopsy, or surgical resection. The histological type was confirmed by pathological morphology and immunohistochemistry. The study was approved by the medical ethics review board of Dazhou Central Hospital (Ethical Application Ref: IRB00000002-17002). The medical ethics review 
103

104

105

106

107

108

109

110

111

112

113

114

115

116

117

118

119

120

121

122

123

board waived the need for informed consent from participants in this study.

The exclusion criteria were as follows: (i) patients who lacked serum CEA data (n=7); (ii)

patients diagnosed with lung cancer, but without a pathological histology basis $(n=18)$. Finally,

213 patients were included in the analyses.

Index Test

Serum CEA level was detected at the initial stage of cancer diagnosis, using a CEA test kit

(Roche Diagnostics Corp, China) by cobas e601 analyzer (Werner et al. 2016). The experimental

operation was carried out according to the reagent protocol. The standard cut-off value of CEA

was $5.0 \mathrm{ng} / \mathrm{mL}$, as recommended by the manufacturers of the assay kits.

\section{Definition of groups}

Metastasis group: The presence of metastasis was confirmed within the first period of admission diagnosis (no more than one month). Patients diagnosed with lung cancer and with obvious images of metastasis in imaging were included in the metastasis group.

Non-metastasis group: Patients with lung cancer but without detected any lymph node metastasis, intrapulmonary metastasis, and any other metastasis within the first hospitalization period and metastases detected beyond the first hospitalization period were included in the nonmetastasis group.

Single metastasis group: Single metastasis is for a single organ, not for single site. Patients with a single metastasis site and multiple metastases sites in one single organ were all included in the single metastasis group.

Multiple metastases group: Patients with multiple organ metastases were included in the 
124

125

126

127

128

129

130

131

132

133

134

135

136

137

138

139

140

141

142

143

144

multiple metastases group.

Non-distant metastasis group: Patients with simple mediastinal/hilar lymph nodes and/or intrapulmonary metastasis were included in the non-distant metastasis group.

Distant metastasis group: Patients excluded from the non-distant metastasis group were included in the distant metastasis group.

\section{Reference Standard}

In our study, the diagnosis of metastasis was based on the complete pathological evidence in the diagnosis of lung cancer, combined with the patient's clinical characteristics and imaging evidence (If necessary, this was combined with pathological examination and/or the specific expression level of tumor markers, such as the confirmation of partial lymph node metastasis by

lymph node dissection, the existence of pathological evidence of pleural effusion and pericardial effusion, and the high expression of CA125 in pleural effusion, and other diagnostic criteria for metastasis confirmation.). All metastases, not detected during the first hospitalization period (no more than one month), were not considered to have metastases. CT, PET, surgery, MRI and Fluorodeoxyglucose (FDG)-PET-CT scan are common imaging modalities for metastasis assessment (Reck et al. 2014).

\section{Statistical Analysis}

Continuous variables were expressed as median (IQR), while categorical variables were expressed with count and percentage. The Kruskal-Wallis test was used to compare the age difference between patients with and without metastases. The Pearson Chi-squared test was used to compare gender, histology and size of tumor difference between patients with and without 
metastases. The Mann-Whitney U test was used to compare CEA levels between patient groups.

146 The relation between CEA level and metastasis was visualized using restricted cubic spline.

147 Receiver operating characteristic (ROC) curve and area under the curve (AUC) were used to

148 represent the overall test accuracy. The optimal cut-off value was determined using Youden's

149 Index. Sensitivity and specificity of the index test were reported along with the Clopper-Pearson

150 confidence intervals. Covariate adjusted ROC curve was generated using a percentile values

151 approach (Janes \& Pepe 2009). MedCalc and R (version: R 3.4.3 for windows (x64),

152 https://www.r-project.org/) were used for statistical analysis, and a $p$-value $<0.05$ was considered

153 statistically significant.

\section{Results}

155

156

157

158

159

160

161

162

163

164

165

\section{Demographics}

There were 213 patients eligible for the final analyses based on the exclusion criteria, 170 male and 43 female, with a median age of 62 years (range from 35 to 82 years). A cohort diagram was shown in Fig. 1 and the patients' demographic characteristics were shown in Table

1. The distribution of lung cancer metastasis and partial imaging evidence for metastases were shown in Fig. S1 and Fig. S2.

\section{CEA level in assessment of tumor metastasis}

Among the 213 lung cancer patients with a definite diagnosis of cancer, 128 patients were included in the metastasis group and 85 patients were included in the non-metastasis group (Table 1). Serum CEA (log-transformed) was significantly higher in the metastasis group ( $p<$ 0.001, Fig. 2A-B), and the AUC was 0.724 (95\% confidence interval [CI], 0.654-0.793, Fig. 2C). 
166 We further investigated the relation between CEA level and metastasis without predefined model

167 structure. We found a clear trend of increase in metastasis above $5.0 \mathrm{ng} / \mathrm{mL}$ (the red line in Fig.

$168 \mathrm{~S} 3$ ). The optimal cut-off value is $4.69 \mathrm{ng} / \mathrm{mL}$, which was comparable to the recommended cut-

169 off value $5.0 \mathrm{ng} / \mathrm{mL}$, so we used the recommended cut-off value to calculate the sensitivity and

170 specificity. The sensitivity and specificity of serum CEA were 0.851 (95\% CI, 0.763-0.916) and

0.597 (95\% CI, 0.503-0.686) respectively. Positive predictive value (PPV) and negative

predictive value (NPV) were 62.50\% (95\% CI, 56.87\%-67.81\%) and 83.53\% (95\% CI, 75.37\%-

$17389.37 \%$, Table 2).

174

175

176

177

178

179

180

181

182

183

184

185

\section{CEA level in assessment of tumor metastasis quantity}

Among the 128 patients in the metastasis group, 60 patients were included in the single metastasis group and 68 patients were included in the multiple metastases group. Serum CEA was significantly increased in the multiple metastases group when compared to patients in the single metastasis group ( $p<0.001$, Fig. 3A-B), and the AUC was 0.780 (95\% CI 0.699-0.862, Fig. 3C). The optimal cut-off value is $7.17 \mathrm{ng} / \mathrm{mL}$, and when this value is used, the sensitivity and specificity of serum CEA were 0.765 (95\% CI, 0.646-0.859) and 0.733 (95\% CI, 0.603-0.839), respectively. PPV and NPV were 76.47\% (95\% CI, 67.67\%-83.46\%) and 73.33\% (95\% CI, $63.57 \%-81.25 \%$, Table 2).

\section{CEA level in assessment of location of tumor metastasis}

Among the 128 patients in the metastasis group, 21 patients were included in the nondistant metastasis group and 107 patients were included in as the distant metastasis group. Serum 
186

187

188

189

190

191

192

193

194

195

196

197

198

199

200

201

202

203

204

205

CEA was significantly higher in the distant metastasis group when compared to patients in the non-distant metastasis group ( $p<0.001$, Fig. 4A-B), and the AUC was 0.815 (95\% CI 0.733 0.897, Fig. 4C). The optimal cut-off value is $6.03 \mathrm{ng} / \mathrm{mL}$, and when this value is used, the sensitivity and specificity of serum CEA were 0.692 (95\% CI, 0.595-0.777) and 0.952 (95\% CI, 0.762-0.999), respectively. PPV and NPV were 98.67\% (95\% CI, 91.58\%-99.80\%) and 37.74\% (95\% CI, 31.00\%-44.98\%, Table 2).

\section{Impacts of histology, tumor size, patient age and patient gender on the performance of}

\section{CEA}

In order to evaluate the impacts of histology, tumor size, patient age and patient gender on the performance of CEA, Crude ROC curve and ROC curve adjusted of these factors were performed.

Of the 213 patients diagnosed with lung cancer, 10 cases could not be confirmed with histology due to insufficient tissue material for histological diagnoses, while the other 203 patients had definite histological diagnosis (Table 1). In all patients with identified histology, there were $38 \operatorname{SCLC}(38 / 203,18.7 \%)$ and $165 \operatorname{NSCLC}(165 / 203,81.3 \%)$. After adjusting for histological diagnosis, the AUC did not change, thus histological diagnosis has no impact on the assessment performance of CEA (Fig. S4A).Tumor size was determined according to the greatest dimension of tumor. Because of special circumstances, such as emphysema, voluminous pleural effusion, tumor of bronchus, the size of the tumor in 58 patients (out of 213 patients) could not be accurately measured (showed in Table 1), thus leaving 155 patients eligible for this analysis. 
After adjusting for tumor size, the AUC did not change, thus tumor size has no impact on the

207

208

209

210

211

212

213

214

215

216

217

218

219

220

221

222

223

224

225

226

assessment performance of CEA (Fig. S4B). Scatter plot of tumor size and CEA levels also

showed that with the increase of the size of tumors, the increased risk of CEA did not increase

(Multiple $\mathrm{R}^{2}=0.001$, Adjusted $\mathrm{R}^{2}=-0.005, p$-value $=0.68$, Fig. S5). All patients had their age and gender recorded upon receiving the CEA test, and 213 patients were included for this analysis.

After adjusting for the age and gender of the patient, the AUC did not change, thus the age and gender of the patient has no impact on the assessment performance of CEA (Fig. S4C-D).

\section{Discussion}

In this retrospective study, we identified serum CEA as a diagnostic marker for assessing tumor metastasis in newly diagnosed lung cancer patients. Patients in the metastasis group had a significant higher CEA level versus patients in the non-metastasis group. The ROC curve demonstrated that CEA had good ability in assessing tumor metastasis. The optimal cut-off value for CEA in this dataset was $4.69 \mathrm{ng} / \mathrm{mL}$ determined by Youden's Index, which was close to the standard cut-off value $(5.0 \mathrm{ng} / \mathrm{mL})$ supported by CEA test kit. Moreover, in patients with tumor metastasis, serum CEA was significantly increased in patients with multiple metastases compared with patients with single metastasis. A cut-off of $7.17 \mathrm{ng} / \mathrm{mL}$ could optimally distinguish between single metastasis and multiple metastases. Additionally, our data showed that CEA was also an effective marker for other metastases assessment except mediastinal/hilar lymph nodes and intrapulmonary metastases with an optimal cut-off of $6.03 \mathrm{ng} / \mathrm{mL}$ for this purpose. Tumor size, subtypes, patients' age and patients' gender didn't have any impact on the diagnostic performance of serum CEA levels in assessing metastasis. These results suggested 
227 that serum CEA was a valuable marker in assessing tumor metastasis in lung cancer patients.

228 Tumor metastasis, commonly occurred at stage IV, is responsible for majority of lung

229 carcinoma death. Within the different types of lung carcinomas, there is also a preferential

230 metastatic location, such as liver metastasis in SCLC and adenocarcinoma (Shin et al. 2014;

231 Tamura et al. 2015). Most postoperative recurrences of NSCLC are distant metastasis, especially

232 for brain metastasis and bone metastasis. Regardless of the subtype of lung cancer in the patient,

233 the location and quantity of metastasis have a great impact on the selection of clinical treatment

234 options and early intervention. Previous studies have reported that high levels of serum CEA or

235 cerebrospinal fluid CEA are closely related to brain metastasis of lung cancer because of the

236 capacity of CEA to penetrate the blood-brain barrier, behaving in a similar manner to

237 immunoglobulins due to their homologous molecular weights. Similarly, studies also indicated

238 that the expression of serum CEA is associated with bone metastasis in NSCLC (Li et al. 2016;

239 Noris-Garcia \& Escobar-Perez 2004; Reiber 2001; Reiber et al. 1986). Our findings also paid

240 attention to the significant increase of serum CEA in lung cancer patients with multiple organ

241 metastases. In addition, patients with distant metastasis showed notably higher CEA expression

242 compared with mediastinal/hilar lymph node metastasis and intrapulmonary metastasis patients.

243 Treatment and long-term outcomes depend on the type, stage, and general condition of cancer.

244 Common treatments include surgery, chemotherapy and radiotherapy. NSCLC is sometimes

245 treated by surgery, while SCLC usually has a better response to chemotherapy and radiotherapy.

246 Early identification of the presence and location of metastasis is helpful for clinical selection of

247 appropriate treatment options, such as minimally invasive surgery, palliative surgery,

Peer] reviewing PDF | (2019:03:36134:1:1:NEW 23 Jun 2019) 
248 radiotherapy and bone modifier therapy can create better prognosis thus benefiting patients

249 (Reck et al. 2014). High costs of examination, lack of obvious clinical symptoms and other

250 factors can make it difficult to perform comprehensive examinations (such as whole-body

251 imaging) to find and confirm the presence of metastases. But efforts to timely and

252 comprehensively identify tumor metastasis in lung cancer patients would help patients choose

253 rational therapies and prolong the survival of patients. Therefore, our study suggested the serum

254 CEA as a valuable marker for tumor metastases assessment in newly diagnosed lung cancer

255 patients, which could have some implications in clinical application. If elevated serum CEA is

256 found in newly admitted patients with highly suspected lung cancer, more detailed clinical

257 diagnostic measures should be actively sought to improve the timeliness and comprehensiveness

258 of the detection of metastasis.

259 Limitations

260 Several limitations in our study should be acknowledged. Firstly, it was a retrospective-

261 design single center study, which may make the results less generalization. Secondly, the time

262 period of patients retrospectively reviewed was only one year, and the sample size was relatively

263 small.

264 Conclusions

265 Our study revealed the significant qualitative difference of serum CEA in lung cancer

patients with or without tumor metastasis and evaluated the diagnostic performance of CEA testing to determine tumor metastasis. In metastasized cases, patients with multiple metastases 
269 patients showed significantly higher CEA compared with non-distant metastasis cases.

270 Furthermore, tumor size, subtypes, patients' age and gender have no impact on the performance

271 of CEA testing. Taken together, serum CEA has a positive clinical application value in combined

272 with existed techniques for diagnosis of cancer metastasis to timely and comprehensively detect

273 metastasis.

274 
275

276

277

278

279

280

281

282

283

284

285

286

287

288

289

290

291

292

293

294

295

296

297

298

299

300

301

302

303

304

305

306

307

308

309

310

311

312

313

314

\section{References}

Ardizzoni A, Cafferata MA, Tiseo M, Filiberti R, Marroni P, Grossi F, and Paganuzzi M. 2006. Decline in serum carcinoembryonic antigen and cytokeratin 19 fragment during chemotherapy predicts objective response and survival in patients with advanced nonsmall cell lung cancer. Cancer 107:2842-2849.

Arrieta O, Saavedra-Perez D, Kuri R, Aviles-Salas A, Martinez L, Mendoza-Posada D, Castillo P, Astorga A, Guzman E, and De la Garza J. 2009. Brain metastasis development and poor survival associated with carcinoembryonic antigen (CEA) level in advanced non-small cell lung cancer: a prospective analysis. BMC Cancer 9:119.

Arrieta O, Villarreal-Garza C, Martinez-Barrera L, Morales M, Dorantes-Gallareta Y, Pena-Curiel O, Contreras-Reyes S, Macedo-Perez EO, and Alatorre-Alexander J. 2013. Usefulness of serum carcinoembryonic antigen (CEA) in evaluating response to chemotherapy in patients with advanced non small-cell lung cancer: a prospective cohort study. BMC Cancer 13:254.

Beauchemin N, and Arabzadeh A. 2013. Carcinoembryonic antigen-related cell adhesion molecules (CEACAMs) in cancer progression and metastasis. Cancer Metastasis Rev 32:643-671.

Cedres S, Nunez I, Longo M, Martinez P, Checa E, Torrejon D, and Felip E. 2011. Serum tumor markers CEA, CYFRA21-1, and CA-125 are associated with worse prognosis in advanced non-small-cell lung cancer (NSCLC). Clin Lung Cancer 12:172-179.

Crone-Munzebrock W, and Carl UM. 1990. Dual-energy CT-scan quantification of recalcification in osteolyses of the vertebral body due to mammary carcinomas in the course of antineoplastic treatment. Clin Exp Metastasis 8:173-179.

Gaga M, Powell CA, Schraufnagel DE, Schonfeld N, Rabe K, Hill NS, Sculier JP, and Cancer AETFotRotPitMoL. 2013. An official American Thoracic Society/European Respiratory Society statement: the role of the pulmonologist in the diagnosis and management of lung cancer. Am J Respir Crit Care Med 188:503-507.

Janes H, and Pepe MS. 2009. Adjusting for covariate effects on classification accuracy using the covariate-adjusted receiver operating characteristic curve. Biometrika 96:371-382.

Langer CJ, Besse B, Gualberto A, Brambilla E, and Soria JC. 2010. The evolving role of histology in the management of advanced non-small-cell lung cancer. J Clin Oncol 28:5311-5320.

Li BT, Lou E, Hsu M, Yu HA, Naidoo J, Zauderer MG, Sima C, Johnson ML, Daras M, DeAngelis LM, Fleisher M, Kris MG, and Azzoli CG. 2016. Serum Biomarkers Associated with Clinical Outcomes Fail to Predict Brain Metastases in Patients with Stage IV Non-Small Cell Lung Cancers. PLoS One 11:e0146063.

Noris-Garcia E, and Escobar-Perez X. 2004. [Brain metastasis and the carcinoembryonic antigen]. Rev Neurol 38:267-270.

Patz EF, Jr., Campa MJ, Gottlin EB, Kusmartseva I, Guan XR, and Herndon JE, 2nd. 2007. Panel of serum biomarkers for the diagnosis of lung cancer. J Clin Oncol 25:5578-5583.

Reck M, Popat S, Reinmuth N, De Ruysscher D, Kerr KM, Peters S, and Group EGW. 2014. Metastatic non-small-cell lung cancer (NSCLC): ESMO Clinical Practice Guidelines for diagnosis, treatment and follow-up. Ann Oncol 25 Suppl 3:iii27-39.

Reiber H. 2001. Dynamics of brain-derived proteins in cerebrospinal fluid. Clin Chim Acta 310:173-186.

Reiber H, Jacobi C, and Felgenhauer K. 1986. Sensitive quantitation of carcinoembryonic antigen in cerebrospinal fluid and its barrier-dependent differentiation. Clin Chim Acta 156:259-269.

Peer) reviewing PDF | (2019:03:36134:1:1:NEW 23 Jun 2019) 
315

316

317

318

319

320

321

322

323

324

325

326

327

328

329

330

331

332

333

334

335

336

337

338

339
Shin DY, Na, II, Kim CH, Park S, Baek H, and Yang SH. 2014. EGFR mutation and brain metastasis in pulmonary adenocarcinomas. J Thorac Oncol 9:195-199.

Tamura T, Kurishima K, Nakazawa K, Kagohashi K, Ishikawa H, Satoh H, and Hizawa N. 2015. Specific organ metastases and survival in metastatic non-small-cell lung cancer. Mol Clin Oncol 3:217-221.

Torre LA, Bray F, Siegel RL, Ferlay J, Lortet-Tieulent J, and Jemal A. 2015. Global cancer statistics, 2012. CA Cancer J Clin 65:87-108.

Tsoukalas N, Kostakis ID, Giaginis C, Tolia M, Galanopoulos M, Kiakou M, Aravantinou-Fatorou E, Tsapakidis K, Baxevanos P, Litos I, Tzouda V, Tzovaras A, Kyrgias G, Tsiambas E, and Theocharis S. 2017. Carcinoembryonic antigen and carbohydrate antigen 19-9 serum levels in non-small cell lung cancer. $J$ BUON 22:1390-1394.

Wang W, Bian C, Xia D, He JX, Hai P, Zhao R, and Wang YY. 2017. Combining Carcinoembryonic Antigen and Platelet to Lymphocyte Ratio to Predict Brain Metastasis of Resected Lung Adenocarcinoma Patients. Biomed Res Int 2017:8076384.

Wang W, Song Z, and Zhang Y. 2018. Efficacy of brain radiotherapy plus EGFR-TKI for EGFR-mutated non-small cell lung cancer patients who develop brain metastasis. Arch Med Sci 14:1298-1307.

Werner S, Krause F, Rolny V, Strobl M, Morgenstern D, Datz C, Chen H, and Brenner H. 2016. Evaluation of a 5Marker Blood Test for Colorectal Cancer Early Detection in a Colorectal Cancer Screening Setting. Clin Cancer Res 22:1725-1733.

Wood SL, Pernemalm M, Crosbie PA, and Whetton AD. 2014. The role of the tumor-microenvironment in lung cancer-metastasis and its relationship to potential therapeutic targets. Cancer Treat Rev 40:558-566.

Zhang L, Liu D, Li L, Pu D, Zhou P, Jing Y, Yu H, Wang Y, Zhu Y, He Y, Li Y, Zhao S, Qiu Z, and Li W. 2017. The important role of circulating CYFRA21-1 in metastasis diagnosis and prognostic value compared with carcinoembryonic antigen and neuron-specific enolase in lung cancer patients. BMC Cancer 17:96.

Zheng M. 2016. Classification and Pathology of Lung Cancer. Surg Oncol Clin N Am 25:447-468. 
Table $\mathbf{1}$ (on next page)

Patients' characteristics. 


\begin{tabular}{|c|c|c|c|c|}
\hline Characters & $\begin{array}{l}\text { Lung Cancer } \\
\text { (overall) }\end{array}$ & $\begin{array}{c}\text { Lung Cancer } \\
\text { (with metastasis) }\end{array}$ & $\begin{array}{c}\text { Lung Cancer } \\
\text { (without metastasis) }\end{array}$ & $p$-value \\
\hline $\mathbf{N}$ & 213 & 128 & 85 & \\
\hline Age (median(IQR)) & $62.0(53.0,68.0)$ & $61.0(51.0,68.0)$ & $63.0(56.0,68.0)$ & 0.070 \\
\hline Gender & & & & 0.048 \\
\hline Female & $43(20.2 \%)$ & $32(25 \%)$ & $11(12.9 \%)$ & \\
\hline Male & $170(79.8 \%)$ & $96(75 \%)$ & $74(87.1 \%)$ & \\
\hline Histology & & & & 0.713 \\
\hline NSCLC & $165(77.5 \%)$ & $97(75.8 \%)$ & $68(80 \%)$ & \\
\hline Adenocarcinoma & $64(30 \%)$ & $49(38.3 \%)$ & $15(17.6 \%)$ & \\
\hline Squamous cell & $100(46.9 \%)$ & $47(36.7 \%)$ & $53(62.4 \%)$ & \\
\hline Large Cell & $1(0.5 \%)$ & $1(0.8 \%)$ & $0(0 \%)$ & \\
\hline SCLC & $38(17.8 \%)$ & $24(18.8 \%)$ & $14(16.5 \%)$ & \\
\hline Unclassified & $10(4.7 \%)$ & $7(5.5 \%)$ & $3(3.5 \%)$ & \\
\hline Size of tumor $(\mathrm{cm})$ & & & & 0.401 \\
\hline$<=3$ & $34(16 \%)$ & $23(18 \%)$ & $11(12.9 \%)$ & \\
\hline $3-5$ & $60(28.2 \%)$ & $33(25.8 \%)$ & $27(31.8 \%)$ & \\
\hline $5-7$ & $41(19.2 \%)$ & $21(16.4 \%)$ & $20(23.5 \%)$ & \\
\hline$>7$ & $20(9.4 \%)$ & $12(9.4 \%)$ & $8(9.4 \%)$ & \\
\hline Unmeasured & $58(27.2 \%)$ & $39(30.5 \%)$ & $19(22.4 \%)$ & \\
\hline
\end{tabular}

1 Abbreviations: NSCLC, Non-small cell lung cancer; SCLC, Small Cell Lung Cancer.

2 Unclassified: The pathologic diagnosis was lung cancer, but without typing.

3 Unmeasured: Because of special circumstances, such as emphysema, voluminous pleural effusion, tumor of

4 bronchus, the size of the tumor could not be accurately measured. 
Table 2 (on next page)

Diagnostic performance of CEA. 


\begin{tabular}{lccc}
\hline Outcome & $\begin{array}{c}\text { Metastasis } \\
\text { (all) }\end{array}$ & $\begin{array}{c}\text { Number of tumors } \\
\text { (in metastasis patients) }\end{array}$ & $\begin{array}{c}\text { location of metastasis } \\
\text { (in metastasis patients) }\end{array}$ \\
\hline N & 213 & 128 & 128 \\
AUC (95\% CI) & $0.724(0.654-0.793)$ & $0.780(0.699-0.862)$ & $0.815(0.733-0.897)$ \\
Cut-off (in ng/mL) & 5.0 & 7.17 & 6.03 \\
Sensitivity (95\% CI) & $85.11 \%(76.28 \%, 91.61 \%)$ & $76.47 \%(64.62 \%, 85.91 \%)$ & $69.16 \%(59.50 \%, 77.73 \%)$ \\
Specificity (95\% CI) & $59.66 \%(50.28 \%, 68.55 \%)$ & $73.33 \%(60.34 \%, 83.93 \%)$ & $95.24 \%(76.18 \%, 99.88 \%)$ \\
PPV (95\% CI) & $62.50 \%(56.87 \%, 67.81 \%)$ & $76.47 \%(67.67 \%, 83.46 \%)$ & $98.67 \%(91.58 \%, 99.80 \%)$ \\
NPV (95\% CI) & $83.53 \%(75.37 \%, 89.37 \%)$ & $73.33 \%(63.57 \%, 81.25 \%)$ & $37.74 \%(31.00 \%, 44.98 \%)$ \\
\hline
\end{tabular}

1Abbreviations: AUC, Area Under Curve; PPV, Positive predictive value; NPV, Negative predictive value; CI, zonfidence interval. 
Figure 1

Cohort diagram of the criteria for patients inclusion and exclusion. Abbreviations: CEA, Carcinoembryonic antigen.

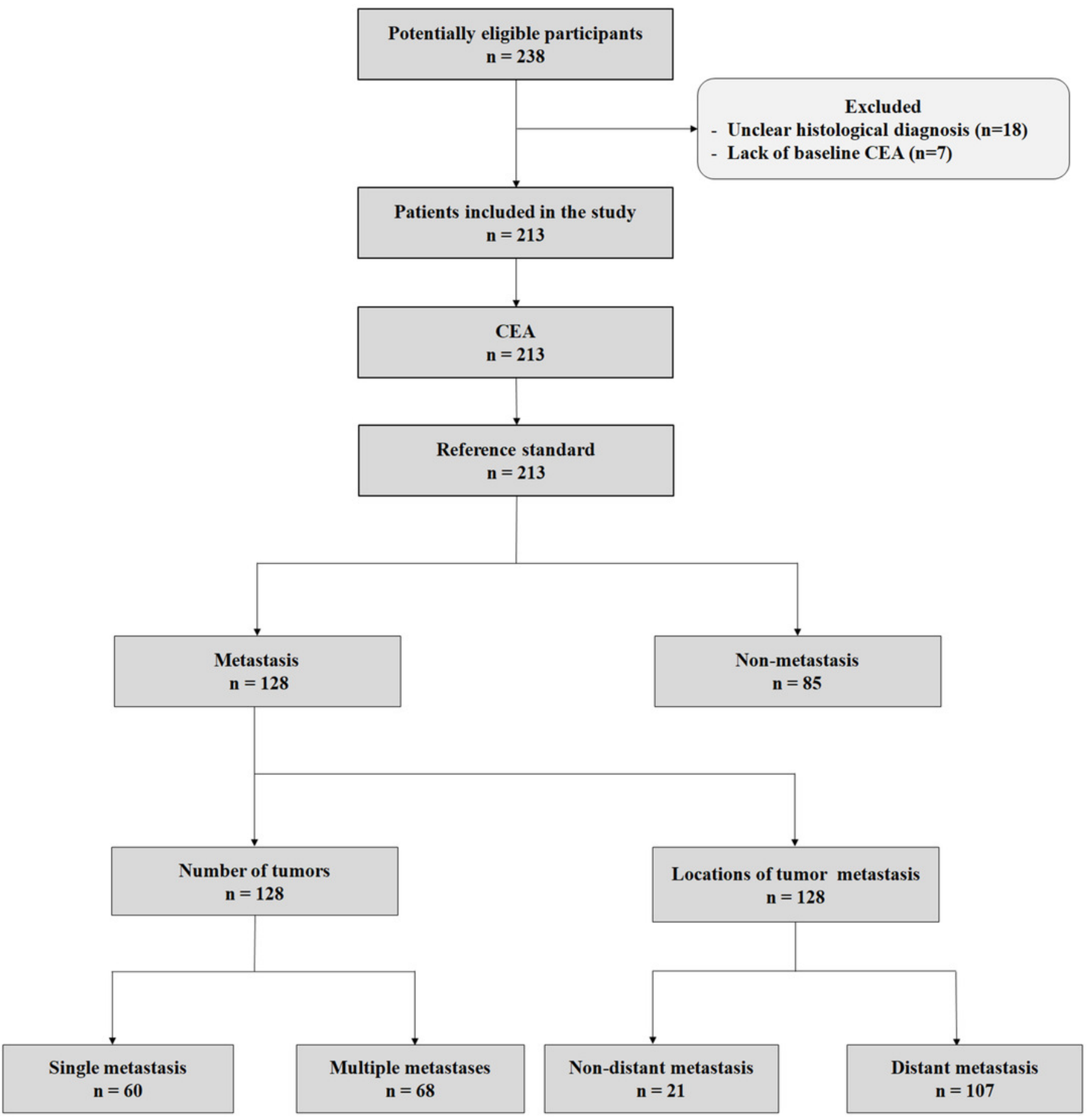


Figure 2

CEA level in diagnosis of tumor metastasis.

'(A) Boxplot of $\log (C E A)$ in Metastatic group and non-metastatic group. (B) Distribution of $\log (C E A)$ grouped by metastasis (yes versus no). (C) Receiving operator characteristic curve $(R O C)$ analysis based on the sensitivity and specificity of CEA on tumor metastasis.'
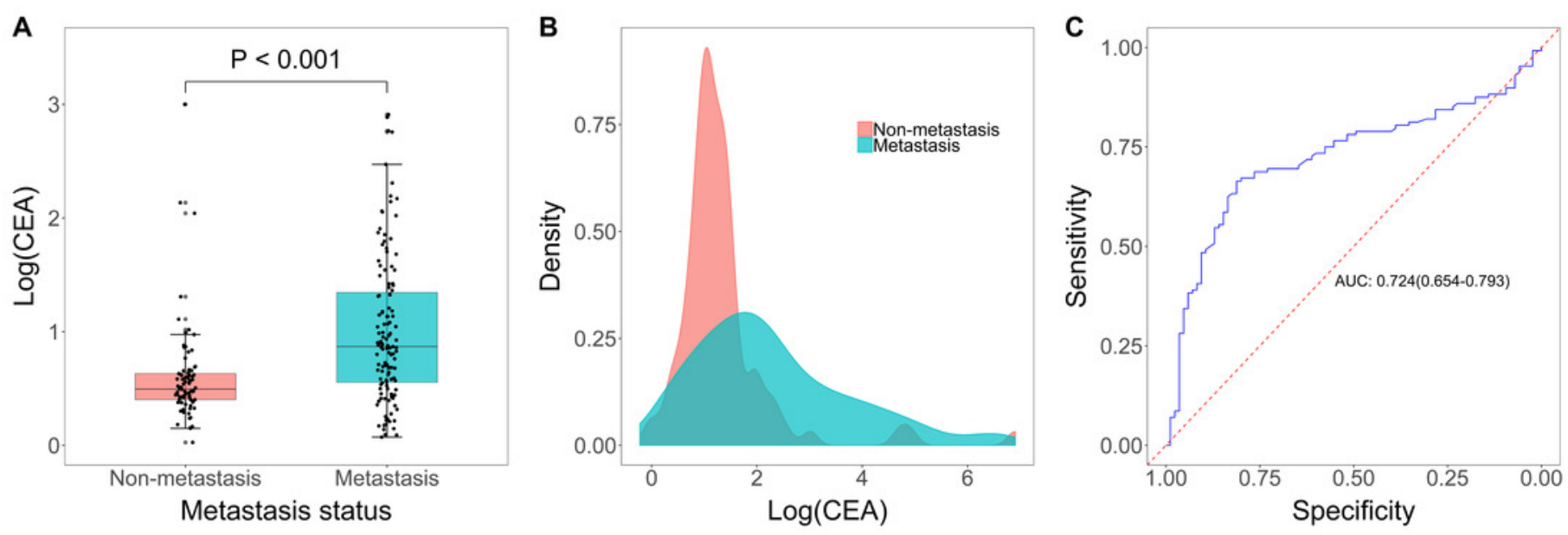
Figure 3

CEA level in diagnosis of tumor metastasis quantity.

'(A) Boxplot of $\log (C E A)$ in single metastasis group and multiple metastases group. (B) Distribution of $\log (C E A)$ grouped by number of tumor metastasis. (C) Receiving operator characteristic curve based on the sensitivity and specificity of CEA on tumor metastasis (1 versus $>=2) . '$
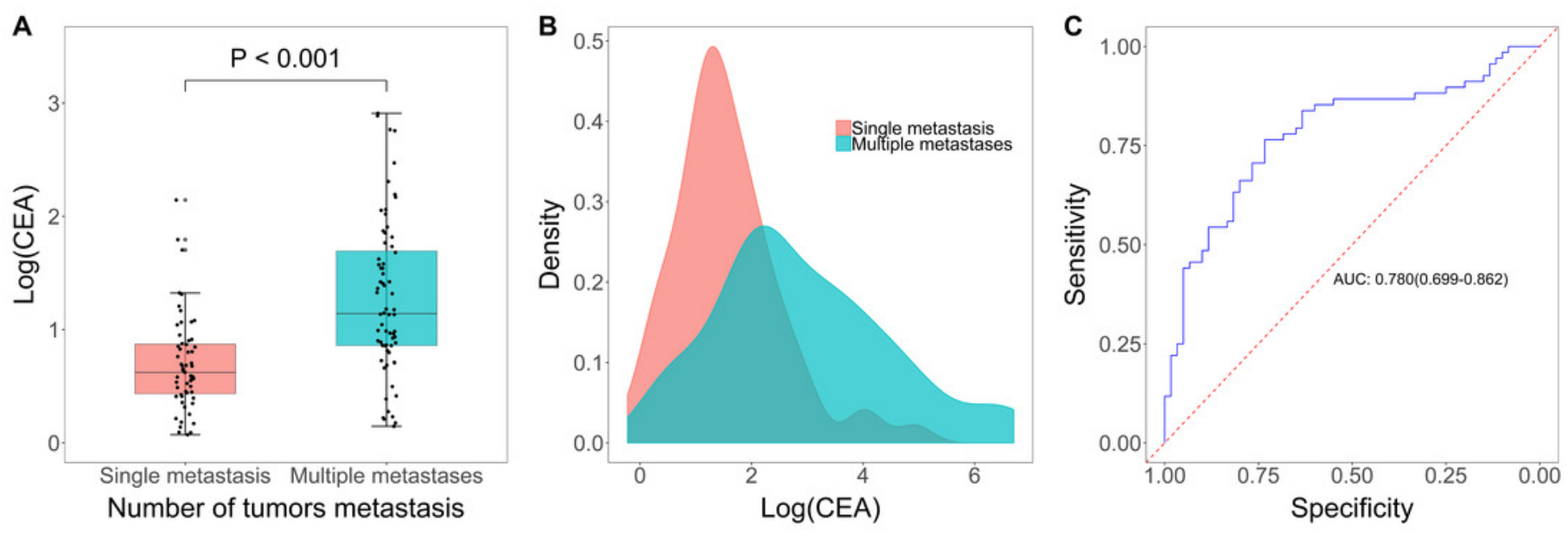
Figure 4

CEA level in diagnosis of location of tumor metastasis.

'(A) Boxplot of $\log (C E A)$ in distant metastasis group and non-distant metastasis group. (B) Distribution of $\log (C E A)$ grouped by location of metastasis. (C) Receiving operator characteristic curve based on the sensitivity and specificity of CEA on location of metastasis (distant versus non-distant).'
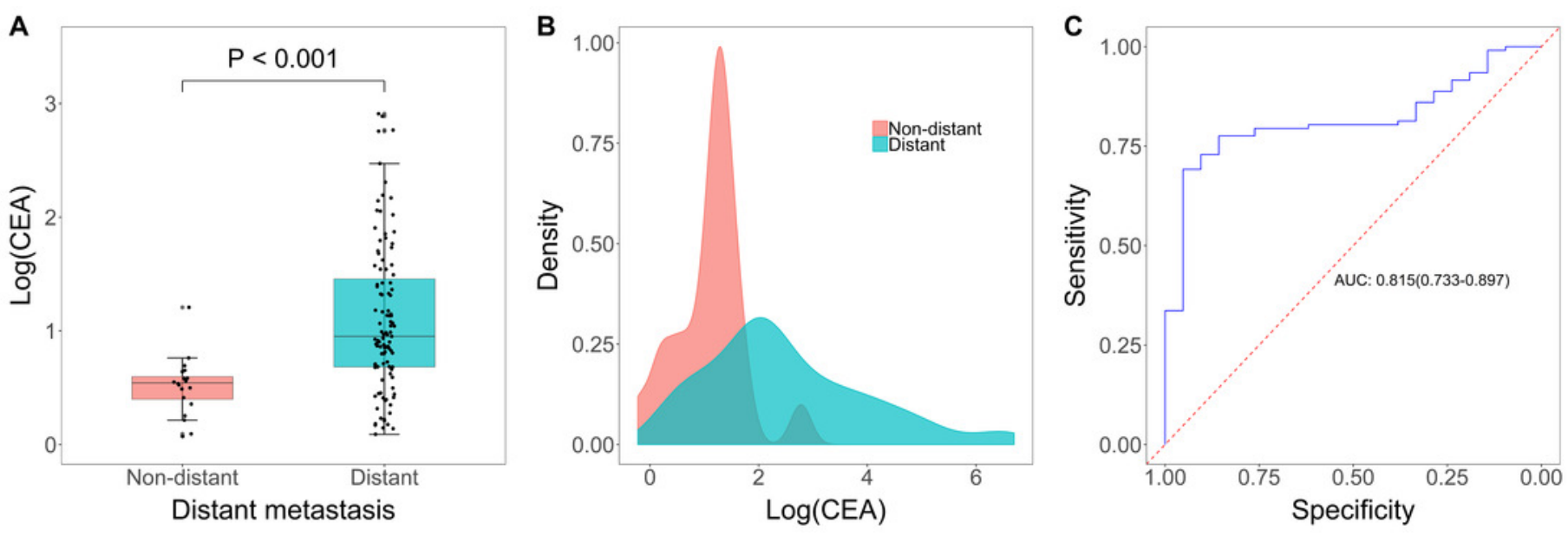